\title{
Tamanho de parcela para produtividade de grãos de sorgo granífero em diferentes densidades de plantas
}

\author{
Sidinei José Lopes ${ }^{(1)}$, Lindolfo Storck ${ }^{(1)}$, Alessandro Dal'Col Lúcio(1), Leandro Homrich Lorentz(1), \\ Cláudio Lovato ${ }^{(1)}$ e Vilnei de Oliveira Dias ${ }^{(1)}$
}

\begin{abstract}
(1)Universidade Federal de Santa Maria, Dep. de Fitotecnia, Avenida Roraima, s/no, Bairro Camobi, CEP 97105-900 Santa Maria, RS. E-mail: sjlopes@pop.com.br, storck@ccr.ufsm.br, adlucio@smail.ufsm.br, llorentz@bol.com.br, clovato@ccr.ufsm.br, vilneid@bol.com.br
\end{abstract}

\begin{abstract}
Resumo - O objetivo deste trabalho foi verificar a influência do arranjo de plantas na estimativa do tamanho ótimo de parcela da cultura de sorgo granífero, para a variável produtividade de grãos. O delineamento estatístico utilizado foi o de blocos ao acaso, num esquema fatorial com dois espaçamentos entre linhas $(0,50 \mathrm{~m}$ e 0,80 m), três densidades de semeadura (100 mil, 160 mil e 220 mil plantas ha $^{-1}$ ) e quatro repetições. Cada repetição foi composta, na área útil, por 12 unidades básicas de 0,50 m da linha de cultivo. Foram ajustados modelos, na estimativa do tamanho ótimo de parcela, que relacionam a variância ou o coeficiente de variação com quatro tamanhos simulados das parcelas. O tamanho estimado de parcelas, na cultura de sorgo granífero, é de 3,2 $\mathrm{m}^{2}$ para a variável produtividade de grãos. $\mathrm{O}$ aumento do número de plantas, na linha, não proporciona incrementos na produtividade de grãos, porém resulta em melhorias da qualidade de experimentos com sorgo. A estimativa do tamanho ótimo de parcela depende do número de plantas utilizadas na unidade básica. O espaçamento entre linhas não influencia na estimativa do tamanho ótimo de parcela.
\end{abstract}

Termos para indexação: Sorghum bicolor, unidade básica, planejamento experimental.

\section{Experimental plot size in grain sorghum in different plant densities}

\begin{abstract}
The objective of this work was to establish plant arrangement effect on the optimal plot size estimates of grain sorghum yield experiments. The experimental design was a completely randomized block with factorial combination of two row spacings ( $0.50 \mathrm{~m}$ and $0.80 \mathrm{~m}$ ), and three plant densities (100 thousand, 160 thousand and 220 thousand plants ha-1), and four replications. The area of each replication was composed by 12 basic units measuring $0.50 \mathrm{~m}$ in row length. Models were adjusted in optimum plot size estimates that correlate variance or variation coefficient with four simulated plot sizes. Plot size was $3.2 \mathrm{~m}^{2}$ for grain sorghum yield experiments. Increasing number of plants in row did not result in higher seed yields, however it improved sorghum quality experiment. Estimation of the ideal plot size depends on number of plants used in the basic unit. Row spacing did not affect estimation of the ideal plot size.
\end{abstract}

Index terms: Sorghum bicolor, basic unit, experiment planning.

\section{Introdução}

A produção de sorgo no Brasil tem aumentado nos últimos anos, tanto pela expansão da área plantada, quanto pelos acréscimos de produtividade (Silva \& Almeida, 2004). As determinações da densidade de semeadura e espaçamentos entre linhas ideais para diversas situações de manejo da cultura, entre outros fatores, são primordiais para otimizar a produtividade (Berenguer \& Faci, 2001; Hammer \& Broad, 2003).

As unidades experimentais, ou parcelas dos experimentos, devem sempre apresentar estandes uniformes de plantas, para reduzir o erro experimental, o que normalmente é obtido pela semeadura em excesso, com posterior desbaste para o estande desejado que, conceitualmente, é a população de plantas com maior produtividade e melhor aproveitamento dos recursos e insumos disponíveis (Pereira, 1989).

Os experimentos devem ser planejados, de tal forma que, se existir variação entre tratamentos, esta seja detectada pela análise estatística, portanto, o erro experimental, estimado pelas repetições de um mesmo tratamento, tem que ser mínimo. A estimativa do erro experimental é afetada por diversos fatores, do início ao final da condução de um experimento: do planejamento - com a escolha do tamanho de parcela adequada à variabili- 
dade das unidades experimentais - à análise estatística - com o não-atendimento das pressuposições do modelo matemático (Storck et al., 2000).

O material que compõe os tratamentos também pode afetar a precisão do experimento (Steel et al., 1997). Alguns experimentos têm identificado a magnitude da variabilidade inerente a alguns tipos de tratamentos, e sua associação com o tamanho e forma ideal de parcela. Lopes \& Storck (1998), em experimentos com diferentes adubações em milho, concluíram que existe uma relação entre o tamanho ideal de parcela e o tipo de adubação, em que o tamanho menor de parcela testado $\left(5 \mathrm{~m}^{2}\right)$ apresentou menor variância, no menor nível de adubação química, para produtividades de grãos menores do que $3 \mathrm{t} \mathrm{ha}^{-1}$. Em experimentos de competição de genótipos de milho, foi observado que o tamanho ótimo de parcela varia entre os diferentes genótipos (Martin, 2003). Jaggard (1975) conduziu um ensaio, para determinar o tamanho e forma ótima de parcela para a cultura de beterraba, e concluiu que a utilização de densidades de plantas diferentes não afeta, significativamente, a forma da parcela.

A preocupação com a eficiência de condução de experimentos, para determinar a população ideal de plantas, existe há muitos anos, o que fez surgir os delineamentos sistemáticos, propostos por Nelder (1962), com a finalidade de melhorar o aproveitamento da área experimental e, conseqüentemente, aumentar o número de tratamentos, o que proporcionaria modelos de regressão mais representativos do comportamento das plantas.

Vários experimentos são realizados para estabelecer a população ideal de plantas para cada genótipo, de modo a proporcionar maior produtividade, em determinada condição experimental. Ainexistência de competição entre plantas vizinhas proporciona produtividade máxima por planta, embora seja baixa por área e, à medida que aumenta a competição intra-específica, há uma relação negativa entre o número de plantas e a produtividade por planta (Pereira, 1989). A produtividade máxima, por área, será obtida com o decréscimo da produtividade por planta compensado pelo maior número de indivíduos. Se o maior número de plantas, por parcela, tem maior precisão na estimativa da média de tratamentos de diferentes populações de plantas, o tamanho de parcela ideal, para esses experimentos, deve corresponder à condição de plantas sem competição intra-específica.

O tamanho de parcela utilizado em experimentos com sorgo granífero, normalmente, segue as informações relativas à cultura do milho, pois não existe na literatura nacional nenhum trabalho específico para determinar o tamanho ideal de parcela para o sorgo.

O objetivo deste trabalho foi verificar a influência do arranjo de plantas nas estimativas do tamanho ótimo de parcela, da cultura de sorgo granífero, para a variável produtividade de grãos.

\section{Material e Métodos}

O experimento foi realizado no ano agrícola 20032004, na Universidade Federal de Santa Maria, Santa Maria, RS. O relevo é plano-ondulado, e o solo é classificado como Brunizem Hidromórfico (Embrapa, 1999). A adubação foi realizada conforme recomendações da Comissão de Fertilidade do Solo - RS/SC (1995). Utilizou-se o sorgo granífero (híbrido AG1018), e as combinações de dois espaçamentos entre linhas $(0,50 \mathrm{~m}$ e $0,80 \mathrm{~m}$ ) e três densidades de semeadura na linha (100 mil, 160 mil e 220 mil plantas ha-1 $^{-1}$ constituíram os tratamentos, em número de seis, repetidos quatro vezes, no delineamento de blocos ao acaso. As repetições de cada tratamento ou unidades experimentais foram compostas por quatro filas, de $4 \mathrm{~m}$ de comprimento, em que as duas filas centrais formaram a área útil para avaliação da produtividade de grãos, excluindo-se 0,50 m nas extremidades. No momento da colheita, a área útil da unidade experimental foi subdividida em 12 unidades básicas, de 0,50 m das linhas de cultivo, totalizando 288 unidades básicas. A semeadura manual foi realizada em 4 de novembro de 2003, colocando-se, aproximadamente, o correspondente a 300 mil sementes ha ${ }^{-1}$, numa profundidade de $5 \mathrm{~cm}$ que, após o desbaste, realizado em 26 de novembro de 2003, resultou nas três densidades em estudo. Os controles de plantas daninhas e irrigações foram realizados, para não serem limitantes ao crescimento e desenvolvimento da cultura.

As panículas de sorgo, colhidas por unidade básica ( $0,50 \mathrm{~m})$, foram embaladas separadamente, com posterior secagem em estufa a $42^{\circ} \mathrm{C}$, por 24 horas. A seguir, foi feita a debulha manual, os grãos foram pesados e $o$ teor de umidade foi medido, para estimativa de produtividade de grãos por hectare, com umidade de $13 \%$.

Utilizando-se as 12 unidades básicas, em cada uma das repetições dos tratamentos, simularam-se diferentes tamanhos de parcela, formados por X1 unidades básicas de comprimento (colunas) e X2 unidades básicas de largura (filas). Portanto, os tamanhos de parcela foram simulados pelo agrupamento de unidades contíguas, de modo que X1xX2 correspondesse a X (tama- 
nho da parcela em número de unidades básicas), que foram: 1x1, 2x1, 3x1 e 6x1. Foram estabelecidos os parâmetros: número de parcelas $(\mathrm{N})$, com $\mathrm{X}$ unidades básicas (UB), de tamanho calculado por $\mathrm{N}=12 / \mathrm{X}$; média das parcelas $[\mathrm{M}(\mathrm{x})]$, com X UB de tamanho; variância entre parcelas $[\mathrm{V}(\mathrm{x})]$ de $\mathrm{X}$ UB de tamanho; variância por UB $[\mathrm{VU}(\mathrm{x})]$, calculada entre as parcelas de $\mathrm{X} U \mathrm{UB}=\mathrm{V}(\mathrm{x}) / \mathrm{X}^{2}$; e coeficiente de variação entre as parcelas $[\mathrm{CV}(\mathrm{x})]$ de $\mathrm{X}$ UB de tamanho.

Após o cálculo dessas estatísticas, foi estimado, para cada uma das 24 parcelas, o índice de heterogeneidade do solo (b), segundo a relação empírica $\mathrm{VU}(\mathrm{x})=\mathrm{V}_{1} / \mathrm{X}^{\mathrm{b}}$ para produtividade de grãos (Smith, 1938). O valor de b foi estimado como um coeficiente de regressão linear, pela logaritmização da função $\mathrm{VU}(\mathrm{x})=\mathrm{V}_{1} / \mathrm{X}^{\mathrm{b}}$ e a estimação ponderada pelos graus de liberdade, associados aos quatro tipos de parcelas simulados (Steel et al., 1997). No modelo, $V_{1}$ é o parâmetro para estimar a variância entre as parcelas de uma UB. Também foram avaliados, de forma semelhante, os parâmetros da função $\mathrm{CV}(\mathrm{x})=\mathrm{A} / \mathrm{X}^{\mathrm{B}}$.

Utilizando-se as estimativas dos parâmetros das funções $\mathrm{VU}(\mathrm{x})=\mathrm{V} 1 / \mathrm{X}^{\mathrm{b}}$ e $\mathrm{CV}(\mathrm{x})=\mathrm{A} / \mathrm{X}^{\mathrm{B}}$, e a média das parcelas com uma UB $\left(\mathrm{M}_{1}\right)$, foram obtidas as estimativas do tamanho ótimo de parcela, pelos métodos de máxima curvatura modificada (MO), máxima curvatura em função da VU (MV) e máxima curvatura em função do CV (MC), respectivamente:

$\mathrm{Xo}=\exp \left\{[1 /(2 \mathrm{~B}+2)] \ln \left[\mathrm{A}^{2} \mathrm{~B}^{2}(2 \mathrm{~B}+1) /(\mathrm{B}+2)\right]\right\}$ (Meier \& Lessman, 1971);

$\mathrm{Xo}=\exp \left\{[1 /(2 \mathrm{~b}+2)] \ln \left[\mathrm{bV1} 1^{2}(2 \mathrm{~b}+1) /(\mathrm{b}+2)\right]\right\}$ (Thomas, 1974); e

$\mathrm{Xo}=\exp \left\{[1 /(\mathrm{b}+2)] \ln \left[\mathrm{b}^{2}(\mathrm{~b}+1) \mathrm{V} 1 /\left(2(\mathrm{~b}+4) \mathrm{M}_{1}^{2}\right)\right]\right\}$ (Thomas, 1974).

As análises foram realizadas com o auxílio dos pacotes estatísticos NTIA/Embrapa e um programa específico em linguagem Fortran, para estimar as variâncias entre as parcelas de diferentes tamanhos. Na comparação das médias de produtividade de grãos, estimadas na unidade básica e por planta, utilizou-se o teste de Tukey a $5 \%$ de probabilidade.

\section{Resultados e Discussão}

Houve disparidade, nos valores estimados, entre o método MO e os métodos MV e MC; MO estima sempre valores superiores aos demais (Tabela 1).

Os métodos MV e MC apresentaram estimativas de tamanho de parcela que não condizem com os resulta- dos normalmente encontrados, e nem com tamanhos de parcela utilizados na prática, o que pode ser constatado no tratamento, com espaçamento de 0,80 m entre linhas e 160 mil plantas ha-1 ${ }^{-1}$ em que a média do tamanho estimado foi de 0,11 unidades básicas, ou seja, $0,02 \mathrm{~m}^{2}$. Os métodos MV e MC têm apresentado resultados inadequados tanto em condição de baixo número de unidades básicas, como é o caso desse experimento, quanto em ensaios com número de unidades básicas relativamente altos, como encontrado em Martin (2003), que utilizou 96 unidades básicas por parcela. Os valores de tamanho de parcela obtidos pelos métodos MV e MC, em sua maioria, ficaram abaixo do tamanho de uma unidade básica e, portanto, foram desconsiderados (Gomez \& Gomez, 1984).

Os índices de heterogeneidade do solo (b), nas repetições dos diferentes tratamentos, tiveram uma amplitude de variação, que foi de uma condição de alta homogeneidade $(b=0,02)$ até alta heterogeneidade (b próximo a 1), isto significa grandes variações na área experimental, entre repetições de um mesmo tratamento, e entre tratamentos. Entre os tratamentos, as variações dos valores obtidos, em relação ao índice de heterogeneidade do solo, não apresentam o mesmo comportamento das estimativas do tamanho de parcela, descritos acima, ou seja, pelas estimativas dos valores de b e do tamanho de parcela, pelo método MO, são obtidos indicativos diferentes da heterogeneidade da área experimental, porém, deve-se ressaltar que a estimativa de $b$ foi obtida pelas variâncias de quatro tamanhos de parcelas.

Os valores de b também ultrapassaram o máximo teórico ( $b=1$ ) em $50 \%$ das repetições dos tratamentos, ou seja, em 12 parcelas. Thomas (1974), quando fez as deduções matemáticas dos pontos de máxima curvatura da função $\mathrm{CV}(\mathrm{x})$ e $\mathrm{VU}(\mathrm{x})$, relata que naqueles casos a correlação entre as parcelas adjacentes é negativa, e que isto se deve à competição entre as unidades básicas. No caso de uma unidade básica ser composta por mais de uma planta, essa afirmação fica difícil de ser compreendida, pois, na área do ensaio em branco, deveria haver competição entre grupos de plantas de unidades básicas vizinhas. Caso fosse uma planta igual a uma unidade básica, então poderia admitir-se que ao lado de cada planta mais competitiva tivesse uma planta de crescimento menor.

A estimativa do coeficiente de variação das parcelas de uma unidade básica de tamanho - valores de A (a 
origem da função $\left.\mathrm{CV}(\mathrm{x})=\mathrm{A} / \mathrm{X}^{\mathrm{B}}\right)$ - também indica alta variação (em média 52,76\%) entre as repetições e tratamentos do experimento. Excetuando o tratamento composto por $0,50 \mathrm{~m}$ entre linhas e $220 \mathrm{mil}^{\mathrm{l}}$ plantas ha ${ }^{-1}$, os valores do coeficiente de variação foram condizentes com as estimativas do tamanho de parcela, pelo método MO; ou seja, os tratamentos com maior coeficiente de variação necessitam de parcelas maiores.

A forma da função, expressa pelo parâmetro B, depende do valor do coeficiente de variação (parâmetro
A) entre as unidades básicas do ensaio em branco, em que os valores de B estão diretamente associados aos valores de A (Tabela 1). Portanto, a definição do tamanho da unidade básica, a ser utilizado no ensaio de uniformidade, também deve ser escolhida de forma criteriosa e, conseqüentemente, as estimativas de tamanho de parcela com resultados de experimentos com tratamentos - em que a unidade básica é obtida por subdivisões das unidades experimentais - não são comparáveis com as obtidas em ensaios de uniformidade,

Tabela 1. Estimativas da produtividade de grãos do sorgo granífero ( $\left.\mathrm{t} \mathrm{ha}{ }^{-1}\right)$ na unidade básica $\left(\mathrm{M}_{1}\right)$ e por planta (g planta-1), dos parâmetros das relações $\mathrm{CV}(\mathrm{x})=\mathrm{A} / \mathrm{X}^{\mathrm{B}}$ e $\mathrm{VU}(\mathrm{x})=\mathrm{V}_{1} / \mathrm{X}^{\mathrm{b}}$, e do tamanho ótimo de parcela, usando-se os métodos de máxima curvatura modificada (MO), máxima curvatura em função da VU (MV), e máxima curvatura em função de CV (MC), nas quatro repetições (r), de dois espaçamentos entre linhas e três densidades de semeadura. Santa Maria, RS, 2003/2004(1).

\begin{tabular}{|c|c|c|c|c|c|c|c|c|c|c|}
\hline Repetição & $\mathrm{M}_{1}$ & g planta $^{-1}$ & $\mathrm{~A}$ & $\mathrm{~B}$ & $\mathrm{~V}_{1}$ & $\mathrm{~b}$ & $\mathrm{MO}$ & $\mathrm{MO}\left(\mathrm{m}^{2}\right)$ & MV & $\mathrm{MC}$ \\
\hline & \multicolumn{10}{|c|}{$0,50 \mathrm{~m}$ entre linhas e 100.000 plantas $\mathrm{ha}^{-1}$} \\
\hline 1 & 6,6 & 65,6 & 73,40 & 0,84 & 0,0029 & 1,69 & 9,25 & 2,31 & 0,38 & 0,76 \\
\hline 2 & 6,5 & 64,9 & 19,70 & 0,20 & 0,0002 & 0,40 & 2,60 & 0,65 & 0,03 & 0,06 \\
\hline 3 & 5,1 & 50,9 & 55,80 & 1,18 & 0,0010 & 2,32 & 6,91 & 1,73 & 0,42 & 0,82 \\
\hline 4 & 5,7 & 57,3 & 37,80 & 0,51 & 0,0006 & 1,01 & 6,60 & 1,65 & 0,16 & 0,31 \\
\hline Média & $6,0 \mathrm{a}$ & $59,7 \mathrm{a}$ & 46,68 & 0,68 & 0,0012 & 1,36 & 6,34 & 1,59 & 0,25 & 0,49 \\
\hline & \multicolumn{10}{|c|}{$0,50 \mathrm{~m}$ entre linhas e 160.000 plantas $^{-1}$} \\
\hline 1 & 8,2 & 51,0 & 18,80 & 0,25 & 0,0003 & 0,50 & 2,93 & 0,73 & 0,05 & 0,07 \\
\hline 2 & 6,8 & 42,2 & 25,70 & 0,37 & 0,0004 & 0,74 & 4,62 & 1,16 & 0,09 & 0,16 \\
\hline 3 & 6,8 & 42,7 & 79,10 & 2,17 & 0,0039 & 4,44 & 5,27 & 1,32 & 0,72 & 1,25 \\
\hline 4 & 6,0 & 37,6 & 31,60 & 0,75 & 0,0005 & 1,51 & 5,94 & 1,49 & 0,24 & 0,43 \\
\hline Média & $7,0 \mathrm{a}$ & $43,4 \mathrm{abc}$ & 38,80 & 0,89 & 0,0013 & 1,8 & 4,69 & 1,17 & 0,28 & 0,48 \\
\hline & \multicolumn{10}{|c|}{$0,50 \mathrm{~m}$ entre linhas e 220.000 plantas $^{-1}$} \\
\hline 1 & 7,6 & 34,7 & 18,40 & 0,12 & 0,0002 & 0,23 & 1,60 & 0,40 & 0,02 & 0,02 \\
\hline 2 & 5,4 & 24,7 & 27,00 & 0,37 & 0,0003 & 0,73 & 4,79 & 1,20 & 0,08 & 0,16 \\
\hline 3 & 5,9 & 26,8 & 33,70 & 0,19 & 0,0005 & 0,39 & 3,92 & 0,98 & 0,04 & 0,08 \\
\hline 4 & 7,5 & 34,1 & 148,00 & 2,06 & 0,0152 & 4,08 & 6,73 & 1,68 & 0,79 & 1,49 \\
\hline Média & $6,6 a$ & $30,1 \mathrm{bc}$ & 56,78 & 0,69 & 0,0041 & 1,36 & 4,26 & 1,07 & 0,23 & 0,44 \\
\hline & \multicolumn{10}{|c|}{$0,80 \mathrm{~m}$ entre linhas e 100.000 plantas $\mathrm{ha}^{-1}$} \\
\hline 1 & 2,5 & 25,4 & 69,40 & 0,20 & 0,0010 & 0,41 & 7,42 & 2,97 & 0,06 & 1,16 \\
\hline 2 & 5,1 & 50,5 & 49,60 & 0,51 & 0,0020 & 1,01 & 7,91 & 3,16 & 0,21 & 0,37 \\
\hline 3 & 5,3 & 53,0 & 183,50 & 3,18 & 0,0074 & 4,22 & 4,79 & 1,92 & 0,75 & 1,28 \\
\hline 4 & 6,7 & 67,2 & 86,30 & 2,55 & 0,0116 & 5,21 & 4,76 & 1,90 & 0,83 & 1,32 \\
\hline Média & $4,9 \mathrm{a}$ & $49,0 \mathrm{ab}$ & 97,20 & 1,61 & 0,0055 & 2,71 & 6,22 & 2,49 & 0,46 & 1,03 \\
\hline & \multicolumn{10}{|c|}{$0,80 \mathrm{~m}$ entre linhas e 160.000 plantas $\mathrm{ha}^{-1}$} \\
\hline 1 & 6,2 & 38,9 & 40,10 & 0,66 & 0,0020 & 1,33 & 6,90 & 2,76 & 0,29 & 0,43 \\
\hline 2 & 6,4 & 39,9 & 35,40 & 0,06 & 0,0017 & 0,13 & 1,53 & 0,61 & 0,02 & 0,02 \\
\hline 3 & 5,0 & 31,4 & 22,30 & 0,14 & 0,0004 & 0,27 & 2,17 & 0,87 & 0,02 & 0,04 \\
\hline 4 & 7,8 & 48,9 & 37,40 & 0,23 & 0,0028 & 0,46 & 4,84 & 1,94 & 0,09 & 0,11 \\
\hline Média & $6,4 a$ & $39,8 \mathrm{bc}$ & 33,80 & 0,27 & 0,0017 & 0,55 & 3,86 & 1,54 & 0,11 & 0,15 \\
\hline & \multicolumn{10}{|c|}{$0,80 \mathrm{~m}^{2}$ entre linhas e 220.000 plantas $^{-1}$} \\
\hline 1 & 6,2 & 28,0 & 85,70 & 1,75 & 0,0091 & 3,51 & 6,39 & 2,56 & 0,71 & 1,20 \\
\hline 2 & 6,0 & 27,3 & 34,50 & 0,01 & 0,0014 & 0,02 & 0,25 & 0,10 & 0,01 & 0,01 \\
\hline 3 & 5,5 & 25,0 & 32,10 & 0,17 & 0,0010 & 0,34 & 3,47 & 1,39 & 0,05 & 0,07 \\
\hline 4 & 5,3 & 24,0 & 21,00 & 0,78 & 0,0004 & 1,57 & 4,70 & 1,88 & 0,25 & 0,36 \\
\hline Média & $5,8 \mathrm{a}$ & $26,1 \mathrm{c}$ & 43,33 & 0,68 & 0,0030 & 1,36 & 3,70 & 1,48 & 0,26 & 0,41 \\
\hline Média geral & 6,1 & 41,3 & 52,76 & 0,80 & 0,0028 & 1,52 & 4,85 & 1,56 & 0,26 & 0,50 \\
\hline
\end{tabular}

${ }^{(1)}$ Médias seguidas por letras distintas na coluna, diferem entre si, pelo teste de Tukey a 5\% de probabilidade; $\mathrm{A}$ : origem da função $\mathrm{CV}(\mathrm{x})=\mathrm{A} / \mathrm{X}^{\mathrm{B}}$; B: forma da função $\mathrm{CV}(\mathrm{x})=\mathrm{A} / \mathrm{X}^{\mathrm{B}}$; $\mathrm{V}_{1}$ : origem da função $\mathrm{VU}(\mathrm{x})=\mathrm{V}_{1} / \mathrm{X}^{\mathrm{b}}$; b: índice de heterogeneidade do solo. 
que normalmente utilizam tamanhos de unidade básicas inferiores àqueles.

A partir das médias do tamanho ótimo de parcela, pelo método $\mathrm{MO}$, verifica-se que aumentar o número de plantas na linha, de 5 para 11 plantas $\mathrm{m}^{-1}$ (100 mil para 220 mil plantas ha- ${ }^{-1}$ ), no espaçamento entre linhas de $0,50 \mathrm{~m}$, e de 8 para 17,6 plantas $\mathrm{m}^{-1}$ (100 mil para 220 mil plantas ha ${ }^{-1}$ ), no espaçamento de $0,80 \mathrm{~m}$, resulta na possibilidade de utilizar parcelas menores (Tabela 1), isto é, parcelas com maiores densidades de semeadura aumentariam a precisão do experimento, pela maior representatividade da heterogeneidade do solo. Ao considerar experimentos que estudem o efeito de densidade de semeadura, a premissa, para áreas restritas, de melhorar a precisão utilizando-se parcelas menores do que a ideal, com mais repetições, pode acarretar em não-atendimento da pressuposição de homogeneidade das variâncias dos erros.

O efeito de aumentar-se a precisão, com maiores densidades de semeadura, deve estar associado à maior representatividade. Apesar de trabalharem com a mesma densidade de semeadura em milho, Palomino et al. (2000) relatam que quanto menor é o número de plantas nas parcelas, menor é a confiabilidade da estimativa obtida, o que dificulta as inferências a serem feitas sobre o potencial de seleção de genótipos.

Quanto à produtividade de grãos, os tratamentos não diferiram entre si (Tabela 1), fato considerado normal para a cultura que, efetivamente, compensa a produtividade pelo maior número e peso de grãos por panícula, nas densidades menores, ou seja, altas densidades de semeadura não apresentam vantagens na produção da cultura do sorgo (Berenguer \& Faci, 2001). Confirmando esse resultado, observa-se que a competição intraespecífica entre os tratamentos é significativa, pois a produtividade de grãos por planta é superior na menor densidade de semeadura (100 mil plantas ha-1 ${ }^{-1}$, comparado à maior densidade (220 mil plantas ha $\left.{ }^{-1}\right)$, em ambos os espaçamentos. Associando-se esses resultados com as estimativas de tamanho de parcela, pode-se inferir que, em condições de menor competição intra-específica, são necessárias parcelas maiores na estimativa da produtividade de grãos de sorgo. Considerandose, ainda, que a produtividade de grãos não é influenciada pelas diferentes densidades, devem-se utilizar densidades maiores na melhoria da qualidade dos experimentos.

O espaçamento entre linhas de cultivo do sorgo granífero, de 0,50 m e 0,80 m, não parece interagir com as densidades, na estimativa do tamanho ótimo de par-

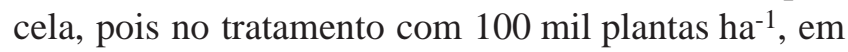
ambos os espaçamentos entre linhas, o tamanho de parcela ficou acima da média geral de 4,85 unidades básicas, e nos tratamentos de 160 mil e 220 mil plantas ha-1, o tamanho ficou abaixo da média geral, independentemente do espaçamento.

Considerando os resultados do método da máxima curvatura modificada, e o maior valor estimado de tamanho de parcela - igual a 7,91 unidades básicas (tratamento com 0,80 m entre linhas e 100 mil plantas ha- ${ }^{-1}$, na repetição 2) -, bem como a adequação prática, podese recomendar o uso de parcelas de $3,2 \mathrm{~m}^{2}$ de área útil, para a variável produtividade de grãos.

\section{Conclusões}

1. O tamanho estimado de parcelas para a cultura do sorgo granífero é de 3,2 $\mathrm{m}^{2}$.

2. Os espaçamentos entre linhas de cultivo do sorgo granífero de 0,50 m e 0,80 m não influenciam na estimativa do tamanho ótimo de parcela.

3. O aumento do número de plantas na linha resulta na necessidade de parcelas menores.

\section{Referências}

BERENGUER, M.J.; FACI, J.M. Sorghum (Sorghum bicolor L. Moench) yield compensation processes under different plant densities and variable water supply. European Journal of Agronomy, v.15, p.43-55, 2001.

COMISSÃO DE FERTILIDADE DO SOLO - RS/SC. Recomendações de adubação e calagem para o estado do Rio Grande do Sul e Santa Catarina. 3.ed. Passo Fundo: SBCC Núcleo Regional Sul; Embrapa/CNPT, 1995. 223p.

EMBRAPA. Centro Nacional de Pesquisa de Solos (Rio de Janeiro, RJ). Sistema brasileiro de classificação dos solos. Brasília: Embrapa-SPI, 1999. 412p.

GOMEZ, K.A.; GOMEZ, A.A. Statistical procedures for agricultural research. $2^{\text {nd }}$ ed. New York: John Wiley, 1984. 680p.

HAMMER, G.L.; BROAD, I.J. Genotype and environment effects on dynamics of harvest index during grain filling in sorghum. Agronomy Journal, v.95, p.199-206, 2003.

JAGGARD, K.W. The size and shape of plots in sugar-beet experiments. Annals of Applied Biology, v.80, p.351-357, 1975.

LOPES, S.J.; STORCK, L. Heterogeneidade do solo sob diferentes adubações na cultura do milho. Ciência Rural, v.28, p.361-366, 1998.

MARTIN, T.N. Contribuição das bases genéticas de milho para o plano experimental. 2003. 86p. Dissertação (Mestrado) Universidade Federal de Santa Maria, Santa Maria. 
MEIER, V.D.; LESSMAN, K.J. Estimation of optimum field plot shape and size for testing yield in Crambe abyssinica Hochst. Crop Science, v.11, p.648-650, 1971.

NELDER, J.A. New kinds of systematic designs for spacing experiments. Biometrics, v.18, p.283-307, 1962.

PALOMINO, E.C.; RAMALHO, M.A.P.; FERREIRA, D.F. Tamanho de amostra para avaliação de famílias de meios-irmãos de milho. Pesquisa Agropecuária Brasileira, v.35, p.14331439, 2000.

PEREIRA, A.R. Competição intra-específica entre plantas cultivadas. O Agronômico, v.41, p.5-11, 1989.

SILVA, A.V.; ALMEIDA, F.A. Cultura do sorgo granífero na Região do Brasil Central. In: CONGRESSO NACIONAL DE MILHO E
SORGO, 25., 2004, Cuiabá. Anais. Cuiabá: Embrapa Gado de Corte; Empaer-MT, 2004.

SMITH, H.F. An empirical law describing heterogeneity in the yields of agricultural crops. Journal of Agricultural Science, v.28, p.123, 1938.

STEEL, R.G.D.; TORRIE, J.H.; DICKEY, D.A. Principles and procedures of statistics. Nova York: McGraw Hill Books, $3^{\text {rd }}$ ed. 1997. 666p.

STORCK, L.; GARCIA, D.C.; LOPES, S.J.; ESTEFANEL, V. Experimentação vegetal. Santa Maria: UFSM, 2000. 198p.

THOMAS, H.L. Relationship between plot size and plot variance. Agricultural Research Journal of Kerala, v.12, p.178-189, 1974.

Recebido em 23 de setembro de 2004 e aprovado em 22 de dezembro de 2004 